\title{
Computational aspects of the through-focus characteristics of the human eye
}

D. Ramos-López, A. Martínez-Finkelshtein and D. R. Iskander

Journal of the Optical Society of America A

2014

This is not the published version of the paper, but a pre-print.

Please follow the links below for the final version and cite this paper as:

D. Ramos-López, A. Martínez-Finkelshtein and D. R. Iskander. Computational aspects of the through-focus characteristics of the human eye. Journal of the Optical Society of America A, Volume 31 (7), Pages 1408-1415, ISSN 1084-7529 (2014).

http://doi.org/10.1364/JOSAA.31.001408

https://www.osapublishing.org/josaa/abstract.cfm?uri=josaa-31-7-1408

(C) [2014, Optical Society of America.]. One print or electronic copy may be made for personal use only. Systematic reproduction and distribution, duplication of any material in this paper for a fee or for commercial purposes, or modifications of the content of this paper are prohibited. 


\title{
Computational aspects of the through-focus characteristics of a human eye
}

\author{
D. Ramos-López, ${ }^{1} \quad$ A. Martínez-Finkelshtein, ${ }^{2}$ and D. Robert Iskander ${ }^{3, *}$ \\ ${ }^{1}$ Department of Mathematics, University of Almería, Almería, Spain \\ ${ }^{2}$ Department of Mathematics, University of Almería, \\ and Institute Carlos I for Theoretical and Computational Physics, Granada, Spain \\ ${ }^{3}$ Institute of Biomedical Engineering and Instrumentation, \\ Wroclaw University of Technology, Wroclaw, Poland
}

\begin{abstract}
Calculating through-focus characteristics of the human eye from a single objective measurement of wavefront aberration can be accomplished through a range of methods that are inherently computationally cumbersome. A simple yet accurate and computationally efficient method is developed, which combines the philosophy of the extended Nijboer-Zernike approach with the radial basis function based approximation of the complex pupil function. The main advantage of the proposed technique is that the increase of the computational cost for a vector valued defocus parameter is practically negligible in comparison to the corresponding scalar valued defocus parameter.
\end{abstract}

OCIS codes: $\quad 330.5370,330.7326,000.3860,000.4430$.

http://doi.org/10.1364/JOSAA.31.001408

\section{INTRODUCTION}

Calculating through-focus characteristics of the human eye's optical system has a wide variety of important applications including assessing the efficacy of intraocular lenses [1-3], studying the depth-of-field and the sensitivity to optical blur [4-6], assessing the role of higher order aberrations [7, 8], assessing the optical changes induced by a refractive surgery [9], and studying the role of retinal image quality in refractive error development [10].

The results of the assessment of the depth-of-field strongly depend on the measurement methodology and whether it is performed subjectively or objectively [5, 11]. Also, the procedures are lengthy, tedious, and require a certain degree of cooperation from the measured subject, as it is the case in the assessment of just detectable image blur. On the other hand, an objectively measured wavefront aberration contains information from which one can derive through-focus characteristics of the eye's optical system through the so-called virtual refraction paradigm $[12,13]$ and derive the dioptric estimate of the depth-of-field using an appropriately selected threshold to a through-focus characteristic function of the retinal image quality $[4,14-16]$.

In the virtual refraction paradigm a through-focus characteristic is sequentially evaluated by adding or

\footnotetext{
* Corresponding author: robert.iskander@pwr.wroc.pl
}

subtracting various amounts of spherical or cylindrical wavefronts to the measured aberration. This can be achieved by either varying the quadratic component of the Zernike polynomial expansion using the dioptric equivalent of defocus [17] or the so-called paraxial curvature matching, which include higher than the second order Zernike polynomial terms [18]. Alternatively, one can employ the concept of Zernike refractive power polynomials $[16,19]$ or the Zernike radial slope polynomials [20] and perform the arythmetics in the refractive power domain. All of those numerical procedures are computationally cumbersome and require substantial computational resources.

Ideally, a closed-form expression of the optical characteristic of the eye's optical system as a function of defocus should be devised. Such attempts have been made with the introduction of the defocus transfer function [21-23] and the extended Nijboer-Zernike (ENZ) approach $[24,25]$. Although both methodologies are attractive for man-made optical systems, they have substantial limitations when it comes to utilizing them to study the eye's optical system because the defocus transfer function is limited to circularly symmetric aberrations while the ENZ approach is valid only for the even terms of Zernike polynomial expansion. The latter is related to the specific way the even-term Zernike polynomial expansion of the wavefront error leads itself to a Bessel-series representation of the diffraction integral. A way to deal with this problem is to seek alternative 
wavefront error representations that, on one hand, fully describe the optical aberrations of the human eye and, on the other hand, would lead to computationally simple mathematical expressions.

To overcome the limitations described above we have developed a technique for calculating through-focus characteristics of the human eye by combining the philosophy of the ENZ approach with the flexibility of the radial basis functions approximation, in which the complex pupil function containing both amplitude apodization and phase wavefront components is described by a series of Gaussian radial basis functions. As a result, we obtain a simple expression for the diffraction integral in terms of a very rapidly converging power series. This allows a parallel implementation of the computation of the through-focus characteristics.

\section{METHODS}

The complex-valued pupil function $P(\rho, \theta)$, which indicates the relative change in amplitude and phase of the light wave transmitted through the pupil, is an essential element in describing the eye's optical system. For a circular pupil, normalized polar coordinates in the pupil plane are used, $0 \leq \rho \leq 1,0 \leq \theta<2 \pi$, in which a pupil function is expressed as

$$
P(\rho, \theta)=A(\rho, \theta) \exp (i \Phi(\rho, \theta)),
$$

where the factor $A(\rho, \theta)$ is the apperture or apodization, and $\Phi(\rho, \theta)$ is the real-valued phase describing the wavefront error. More precisely, function $\Phi(\rho, \theta)$ is equal to the wavefront error $W(\rho, \theta)$ times the constant $2 \pi n / \lambda$, where $n$ is the refractive index and $\lambda$ stands for the wavelength of the light.

According to Fourier optics, the complex-valued point-spread function of such a system is given by the diffraction integral [25]

$$
\begin{aligned}
U(r, \phi ; f) & =\frac{1}{\pi} \int_{0}^{1} \int_{0}^{2 \pi}\left[\exp \left(i f \rho^{2}\right) P(\rho, \theta)\right. \\
& \times \exp ((2 \pi i \rho r \cos (\theta-\phi))] \rho d \theta d \rho
\end{aligned}
$$

where $f$ is the defocus parameter and $(r, \phi)$ denote the polar coordinates in the image plane. This function is also related to the monochromatic point spread function (PSF), defined as $P S F=|U|^{2}$. The defocus parameter $f$ can be related to a wavelength dependent defocus measured in diopters, $D(\lambda)$, as $f=\pi D(\lambda) / 1000 \lambda$, provided that $\rho$ and $\lambda$ are given in $\mathrm{mm}$ [23].

Our main goal is to develop a new approach to an effective (i.e., fast and reliable) computation of these diffraction integrals. It involves approximation by Gaussian radial basis functions (GRBF), which are a standard tool in the solution of a variety of practical purposes, ranging from engineering to numerical analysis of partial derivative equations. Recently, they also found application in the context of ophthalmic optics [26]. A more general version of this type of functions (using the socalled anisotropic radial basis functions) was developed for fitting corneal elevation data [27, 28].
Each GRBF is determined by its center, given by polar coordinates $(q, \alpha)$, and by its shape parameter $L>0$; the expression which evaluates it at a point with polar coordinates $(\rho, \theta)$ is

$$
\operatorname{GRBF}(\rho, \theta)=\exp \left\{-L\left(q^{2}+\rho^{2}-2 \rho q \cos (\theta-\alpha)\right)\right\} .
$$

Thus, as a first step we fix a number of centers, $\left\{\left(q_{k}, \alpha_{k}\right): k=1, \ldots, N\right\},\left(q_{k} \geq 0,0 \leq \alpha_{k} \leq 2 \pi\right)$ and a common value of the shape parameter $L>0$, and fit the complex pupil function (1) by a linear combination of GRBF (see Appendix A), obtaining an expression of the form

$P(\rho, \theta)=\sum_{k=1}^{N} c_{k} \exp \left\{-L\left(q_{k}^{2}+\rho^{2}-2 \rho q_{k} \cos \left(\theta-\alpha_{k}\right)\right)\right\}$

where for each $k=1, \ldots, N, c_{k}$ are complex coefficients. Clearly, it is an approximate formula, but we will continue using the sign "=" instead of " $\approx$ ", assuming that the complex pupil function is actually given by the right hand side of this formula.

By introducing expression (3) in the diffraction integral (2), an analytic closed form expression for $U$ can be obtained in the following way:

$$
U(r, \phi ; f)=\sum_{k=1}^{N} c_{k} U_{k}(r, \phi ; f)
$$

where $U_{k}$ is the contribution of each GRBF to $U$. Simple calculations show that it is explicitly given by

$$
U_{k}(r, \phi ; f)=\exp \left\{-L q_{k}^{2}\right\} \sum_{s=0}^{\infty} \frac{\Omega^{s}}{(s !)^{2}} m_{s}(i f-L)
$$

with

$$
\begin{aligned}
\Omega & =\Omega_{L}\left(r, \phi, q_{k}, \alpha_{k}\right) \\
& =L^{2} q_{k}^{2}+2 \pi i r L q_{k} \cos \left(\phi-\alpha_{k}\right)-\pi^{2} r^{2}
\end{aligned}
$$

and $m_{s}(\xi)$ being the moments of the positive function $e^{\xi \rho}$ on $[0,1]$ :

$$
m_{s}(\xi)=\int_{0}^{1} \rho^{s} e^{\xi \rho} d \rho, \quad s=0,1, \ldots .
$$

Coefficients $m_{s}(\xi)$ satisfy the following recurrence formulas,

$$
m_{0}(\xi)=\frac{e^{\xi}-1}{\xi}, \quad m_{s+1}(\xi)=\frac{e^{\xi}-(s+1) m_{s}(\xi)}{\xi}
$$

Observe that $\Omega$ does not depend on the defocusing parameter $f$, while the sequence $m_{s}(i f-L)$ is independent from the centers $\left(q_{k}, \alpha_{k}\right)$ and evaluation coordinates $(\rho, \theta)$. 
Formulas above can be combined into a single analytic explicit expression for $U$ :

$$
\begin{aligned}
& U(r, \phi ; f)=\sum_{k=1}^{N} c_{k} \exp \left\{-L q_{k}^{2}\right\} \\
& \times \sum_{s=0}^{\infty} \frac{m_{s}(i f-L)}{(s !)^{2}}\left(L^{2} q_{k}^{2}+2 \pi i r L q_{k} \cos \left(\phi-\alpha_{k}\right)-\pi^{2} r^{2}\right)^{s} .
\end{aligned}
$$

By the observation above, coefficients $m_{s}(i f-L)$ need to be evaluated only once, as they do not depend on the individual parameters of each GRBF, and the cost of their computation is independent of the number of functions $N$ in the expansion of the complex pupil function (3). Moreover, due to the presence of the square of a factorial in the denominator, the series in (9) converges very quickly, so that only a small number of terms are needed in practice for its evaluation. Coefficients $m_{s}(i f-L)$ can be efficiently computed in many ways: by definition (7) (using FFT or quadratures) or by the recurrence (8). This last method is very fast, but can show numerical instability for large values of $f$.

The formulas above remain valid if a different shape parameter is taken for each GRBF, i.e., substituting $L$ by $L_{k}$ in the expressions. However, for most practical applications a single value $L$ can be chosen for all GRBF and this assumption has been made for the sake of simplicity.

The final step for the efficient implementation of these formulas is based on the observation that $\Omega_{L}$ in (6) can be easily evaluated by vector-vector multiplication if both the evaluation point and the center of the GRBF are given in the Cartesian coordinates. Moreover, all the computations can be vectorized and parallelized, allowing for a fast evaluation of the diffraction integral simultaneously in a grid of points in the image domain, and for an array of values of the defocus parameter $f$.

\section{COMPARISON OF PROCEDURES}

Since the closed analytic expression for a Fourier transform type integral (2) is possible only for most elementary pupil functions $P$, for its computation we must rely either on numerical or on semi-analytical methods (or analytical approximations). In the first group we find different direct numerical procedures in which the integration over a 2D domain is replaced by evaluation of a discrete sum, with the particular challenge of integrating a highly oscillatory function. In the best known implementation this leads to the bi-dimensional discrete Fourier transform (we will refer to it as the FFT2-based approach), calculated via the Fast Fourier Transform algorithm. Its efficiency can be substantially enhanced using the fractional Fourier transform [29] or a "butterfly diagram" ideas [30], see also [31].

In the second group we can include the so-called Extended Nijboer-Zernike (ENZ) theory [24, 25], based on an observation that the main component of (2) for many
Zernike polynomials can be expressed as series of Bessel functions.

In this section, these alternatives are discussed and compared with our method, paying special attention to their computational complexity, precision, accuracy and speed. We use the "naive" notion of complexity, understanding by this the number of real floating point operations (flops) needed to run the algorithm. Since the exact number of flops is in general difficult or not feasible to calculate, the leading term for large values of the parameters is used.

A comparison between two methods is a delicate task due to their different inherent characteristics. Thus, we tried to make the analysis based on some reasonable assumptions and estimates.

Recall that we want to evaluate $U$ in (2) in a grid of points (either in polar or cartesian coordinates) and for a vector of values of the defocus parameter $f$. All three approaches are inexact in nature and contain some kind of approximation step.

In the FFT2-based scheme, the value of $U(r, \phi ; f)$ is computed by means of the bi-dimensional fast Fourier transform. The crucial step is the substitution of the double integral in (2) by a discrete sum. Additionally, this method presents some other drawbacks. First, each new value of the defocus parameter $f$ obliges to calculate the values of $U$ completely, at a computationally high cost. Second, the use of the FFT requires re-sampling the wavefront at a regular Cartesian grid covering the pupil; for convenience, the length of the grid $M$ should be an integer power of 2 in each direction.

Another remarkable problem of the FFT2 scheme are numerical issues, especially the aliasing. Figure 1 (lower left) shows the aliasing, a typical phenomenon that appears when a careless FFT approach is used. In order to prevent this, the pupil must be small in comparison with the sampled area (or in other words, we must extend the pupil to a larger region, setting the pupil function to zero in the complementary domain), resulting in a large area where $U(r, \phi ; f)$ is negligible [31]. Thus, a big portion of the computational load of this scheme is useless, and in general the spatial resolution needed with this method will be much higher than that required for explicit expressions like (9). This is the common approach used in commercial ray tracing packages, such as Zemax or Code $\mathrm{V}$. The minimal estimated computational complexity of this method for a single value of $f$, even for optimal implementation, is of $\mathcal{O}\left(M^{2} \log (M)\right)$, which corresponds to the cost of the FFT, the most computationally demanding part.

The advantage of the semi-analytic approaches, such as the ENZ theory or the method proposed here, is that they reduce the computation of $U$ in (2) to evaluation of more or less complex explicit expressions in terms of some elementary or special functions. One of the benefits of having these formulas is a better control of the image domain being computed, increasing the precision. However, the real advantage of a formula like (9) or the 

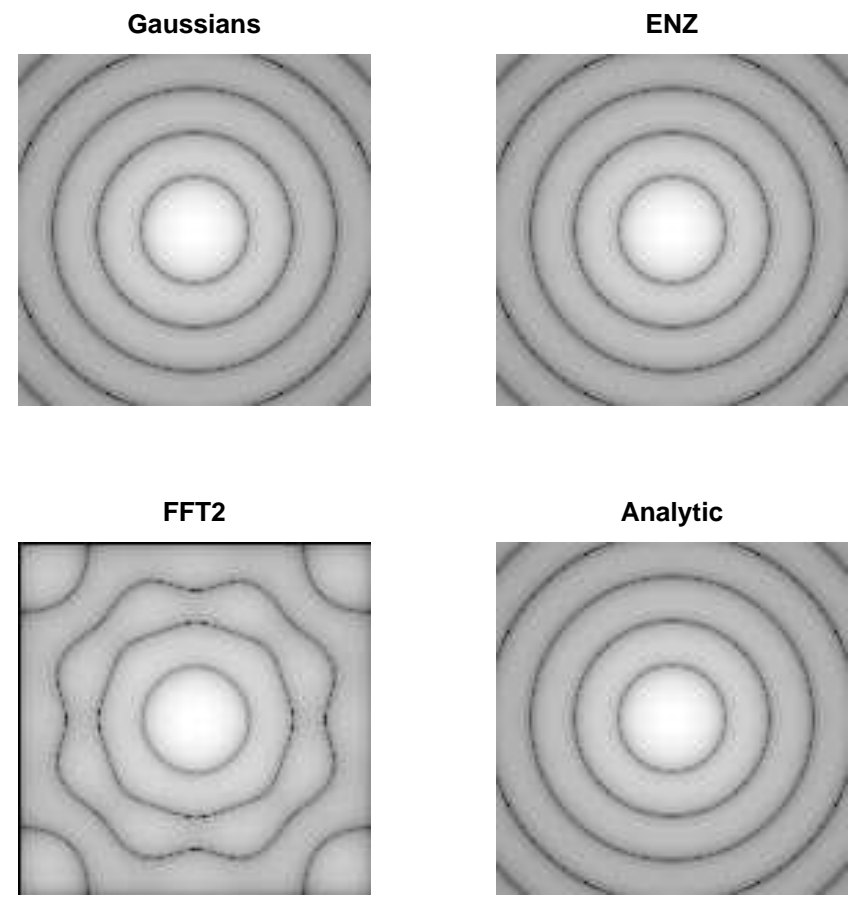

Fig. 1. PSF with perfect wavefront, given by formula (10), calculated by each method.

ENZ-based equivalent is the huge boost in performance gained when a parallelization of calculations is done for multiple values of the defocus parameter $f$.

The ENZ-theory, although representing a big step forward, has some limitations that must be taken into account. The obvious one is the use of only even terms in the Zernike expansion of the complex pupil function, which restricts it to the symmetric wavefront errors. Some other, less evident, problems lie in the core of the mathematical properties of the ENZ explicit formula for $U(r, \phi ; f)$. This is an infinite series of terms, each of them a finite linear combination of Bessel functions, and each new Zernike term added to the expansion of the pupil function (1) increases the complexity of the terms. The series is slowly convergent, especially for larger values of $f$, requiring a truncation with a large number of terms depending on $f$ (it is recommended to use $3|f|+5$ terms, according to $[24,25])$.

Another issue in evaluating the ENZ expressions is the accuracy. The terms of the infinite series with even and odd orders form sign-changing sequences, which increases the risk of the cancellation errors. This phenomenon can be illustrated by the following experiments: in the case when the wavefront is given only by a positive $Z_{2}^{2}$ horizontal astigmatism, the evaluation of, say, the imaginary part of $U$ at a point with radial coordinate $r=0.9$ consists in adding a finite alternating sequence, with two dominant terms of approximately 0.423 , but whose absolute values differ in $3 \times 10^{-5}$. This shows that these calculations, if not well organized, can yield a loss of precision in about 5 significant digits. Last
Table 1. Estimates of the minimal computational complexity of the methods when evaluating $U$ at an $M \times M$ grid of nodes for $F$ values for the defocus parameter $f$, using $N$ functions in the corresponding series expansions (for ENZ and GRBF).

\begin{tabular}{c|c|c} 
Method & Complexity (single $f)$ & Complexity (vector of $f$ ) \\
\hline FFT2 & $\mathcal{O}\left(M^{2} \log (M)\right)$ & $\mathcal{O}\left(F M^{2} \log (M)\right)$ \\
ENZ & $\mathcal{O}\left(M^{2} N+M N^{3 / 2}\right)$ & $\mathcal{O}\left(M^{2} F+M^{2} N F+M N^{3 / 2}\right)$ \\
GRBF & $\mathcal{O}\left(M^{2} N\right)$ & $\mathcal{O}\left(M^{2} F+M^{2} N\right)$
\end{tabular}

but not least, the ENZ formulas contain binomial numbers that must be evaluated with care in order to avoid overflow.

In comparison, in expression (9) based on GRBF an infinite series appears, but its convergence is extremely fast, and only a few terms are required for a precise evaluation. Indeed, since $\left|m_{s}\right| \leq 1$ for all $s$, and also $|\Omega|^{2} \leq L^{2}+\pi^{2} \leq 15$ for any reasonable value of the shape parameter of the Gaussian RBFs. Thus, the $s$ th term in the series in (9) is bounded by $(15)^{s} /(s !)^{2}$, independently of the value of $f$. This shows that, in the worst case scenario, we need at most $N=15$ terms to achieve accuracy of about $10^{-7}$. In practice, the value of $N$ can be taken significantly smaller.

The complexity of the evaluation is also constant for each new Gaussian function added, as they are obtained by the shift of the same base function. We have estimated that with the rest of parameters fixed, the cost of evaluating $U$ with a total of $N$ Zernike polynomials using the ENZ theory grows as $\mathcal{O}\left(N^{3 / 2}\right)$, while for the new scheme with GRBF, with $N$ Gaussian functions, the cost is $\mathcal{O}(N)$.

We summarize the computational cost for each method in Table 1 showing the leading terms in the expression of the estimated complexity. Comparison between rows two and three shows that the GRBF approach is much more efficient than the ENZ theory, especially for a large amount of values for $f$. The FFT2based method seems to be of similar complexity with respect to GRBF, but in practice the value of $M$ for FFT2 will be much larger than that required for GRBF, and the number of functions $N$ will be small (as maximum, 400).

The reader should bear in mind that the complexity estimates give only a rough idea of the computational demand of a method. In general, the execution time is a simpler and a more informative tool. Thus, we run the ENZ and GRBF algorithms evaluating $U$ at an $100 \times 100$ mesh of nodes for a single value of the parameter $f$, recording the execution time in dependence of the value $N$ of functions used in the corresponding series expansions. Figure 2 shows the results, along with the corresponding regression lines. The values of the slopes of these lines are approximately 0.021 seconds/function for ENZ and 0.0028 seconds/function for GRBF. This gives a ratio of about 7.5 times faster for the GRBF approach, with the same number of functions, or reversely, one can 


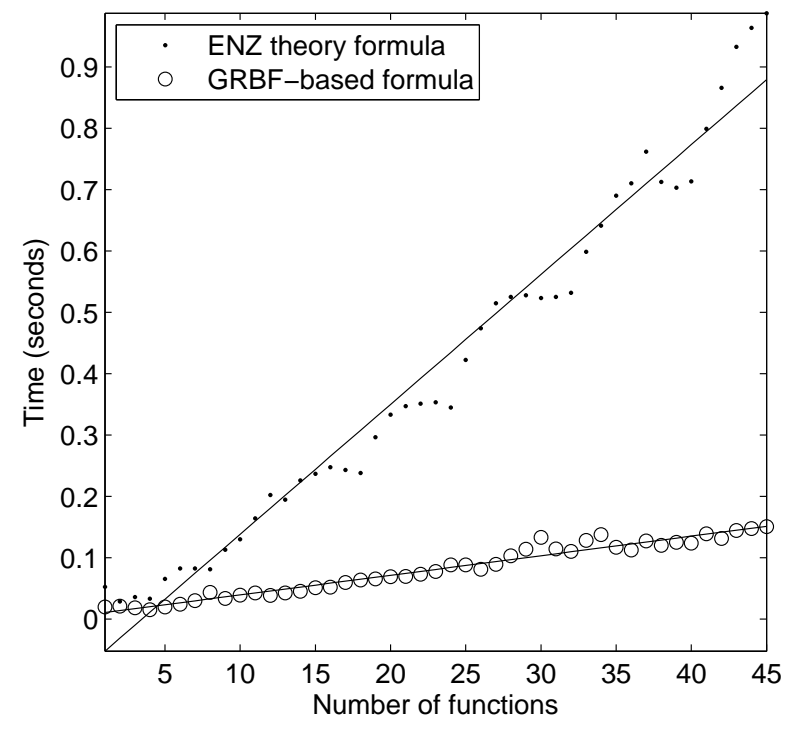

Fig. 2. Dependence of the execution time from the number of functions used in the description of the complex pupil function.

use 7.5 times more functions in the GRBF scheme, for the same execution time. For comparative purposes, the execution time to evaluate function $U$ numerically making use of the two-dimensional fast Fourier transform was of approximately 0.25 seconds, matching the execution time for ENZ using about 12 Zernike terms, or for GRBF with approximately 90 Gaussian RBFs.

In another experiment we compared the execution time of the three methods as a function of the length of the vector of defocus parameters. The number of functions is fixed to $N=400$ for the GRBF approach and to $N=45$ for ENZ-theory (8th order polynomials). The evaluation of $U$ is made at an $100 \times 100$ mesh of nodes for the two semi-analytic methods, and at an $512 \times 512$ mesh for the FFT2-approach (this is a realistic size to overcome aliasing and obtain accurate results). Then, the execution time of each method is measured, as the length of the vector of values for the defocus parameter $f$ grows. The results appear in Figure 3, along with the regression lines for each scheme. The values of the slopes are approximately 0.24 for FFT2, 0.16 for ENZ and 0.003 for GRBF, all in seconds per value of $f$. This means FFT2 is about 75 times slower than GRBF when calculating $U$ for many of values of $f$ at the same time, and ENZ is about 50 times slower than GRBF too, even when the number of Gaussian functions used $(N=400)$ is much higher than the number of Zernike polynomials $(N=45)$.

From the experiments above we can conclude that, roughly speaking, the efficiency of GRBF is at least one order of magnitude higher than of ENZ.

In order to assess accuracy, we first calculated $U$ for the ideal wavefront $(\Phi \equiv 0)$ and zero defocus $(f=0)$, in

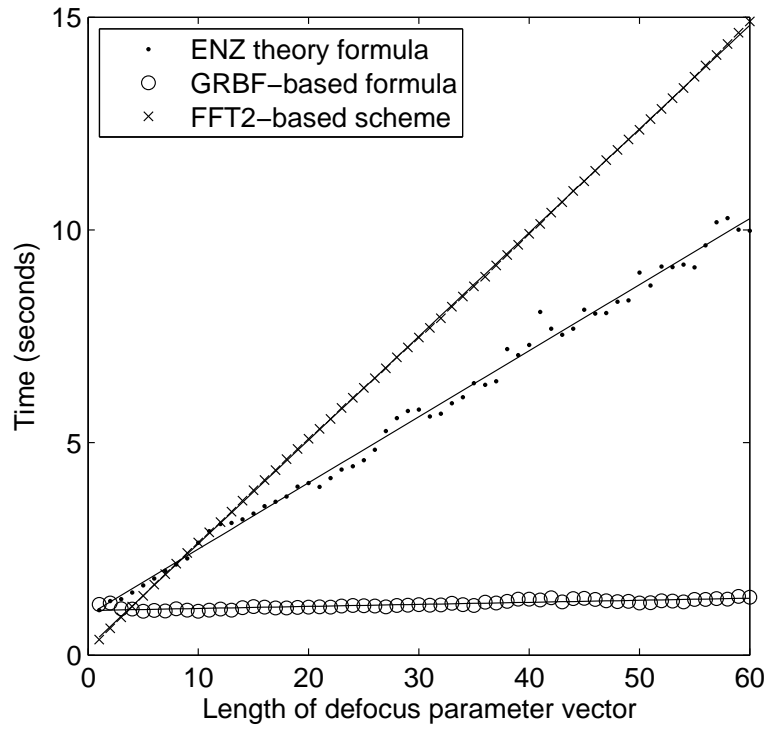

Fig. 3. Execution time according to the number of different defocus parameters used in the complex pupil function. A fixed number $N$ of functions $(N=400$ for GRBF and $N=45$ for ENZ) was used for each value of $f$.

which case the closed analytic expression is known,

$$
U(r, \phi ; 0)=\frac{J_{1}(2 \pi r)}{\pi r},
$$

where $J_{1}$ is the Bessel function [32, Ch. 10]. Figure 1 shows the $P S F=|U|^{2}$ for each of the methods discussed here, as well as the one given by the closed formula. Visually, the two semi-analytic methods perform similarly. In order to support this impression quantitatively, we calculated also the root mean square (RMS) for each approach, which renders approximately $2.5 \times 10^{-8}$ for GBRF, $2.5 \times 10^{-17}$ for ENZ, and $7.6 \times 10^{-3}$ for FFT2. We observe that in this ideal situation the number of accurate digits for GRBF is about a half of those given by ENZ, but still of order of 8 digits, which is usually sufficient for applications. However, GRBF calculations are done at a much lower cost: the computation times were approximately 1.53 seconds for GBRF vs. almost 52 seconds for ENZ. This interval includes obviously both steps: fitting the wave front or the pupil function and the subsequent calculation of $U$ at a grid of $128 \times 128$ nodes.

In another experiment we used a synthetic wavefront (see Figure 4) described by a combination of Zernike polynomial terms and exponentials

$$
\begin{aligned}
\Phi(\rho, \theta)= & -0.5 Z_{4}^{4}(\rho, \theta)+0.3 Z_{5}^{3}(\rho, \theta)+0.3 Z_{5}^{5}(\rho, \theta) \\
& +3 g(\rho \cos \theta, \rho \sin \theta ; 0.5,0.3,5) \\
& +3 g(\rho \cos \theta, \rho \sin \theta ; 0.5,-0.3,5) \\
& +0.2 g(\rho \cos \theta, \rho \sin \theta ;-0.3,0,15),
\end{aligned}
$$

where $Z_{n}^{m}$ are the (orthonormal) Zernike polynomials 


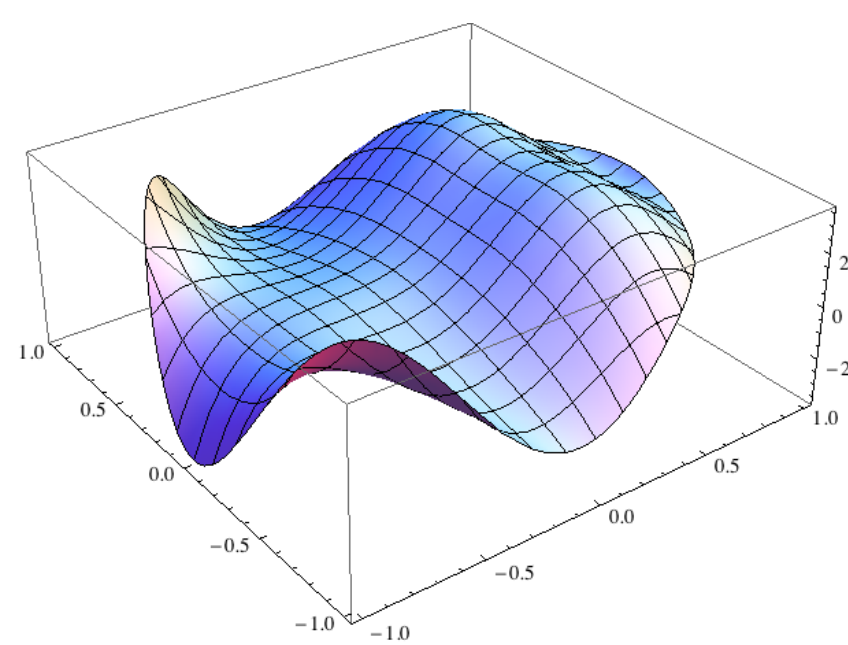

Fig. 4. 3D plot of the synthetic wavefront function defined in (11).

Table 2. Maximum absolute error and root-mean-square error corresponding to the PSF residual distributions shown in Figure 6.

\begin{tabular}{c|c|c|c} 
Metric & GRBF & ENZ & FFT2 \\
\hline Max. error & $5.00 \times 10^{-4}$ & 0.42 & 0.14 \\
RMS error & $6.92 \times 10^{-5}$ & 0.07 & 0.02
\end{tabular}

and

$$
g(x, y ; a, b, L)=\exp \left\{-L\left[(x-a)^{2}+(y-b)^{2}\right]\right\} .
$$

For this wavefront we calculated the diffraction integral (2) for different values of $f$ by quadrature using the scientific software Mathematica with extended precision (with the options PrecisionGoal set to 8 and WorkingPrecision to 16). These values of $U$ (regarded as "exact") were compared with the calculations performed by FFT2 and by two semi-analytic approaches discussed here. For the ENZ we fitted the pupil function using the first 200 Zernike polynomials, while for the GRBF the approximation was performed by the linear combination of $20 \times 20$ Gaussian functions, with the parameter $L=16$. Then the diffraction integral was evaluated by all methods in a grid of $256 \times 256$ equally spaced points in the square $[-2,2] \times[-2,2]$.

In order to assess accuracy, we computed for each method the real-valued point-spread function $P S F=$ $|U|^{2}$ taking first $f=0$. Figure 5 shows the density plot of the normalized PSF calculated by quadrature and using the three alternative methods (FFT2, ENZ and GRBF). The corresponding absolute errors with respect to the computation by quadratures is depicted in Figure 6. More quantitative comparison was made using several quality of approximation metrics, such as those gathered in Table 2.

With the purpose of comparing performance of the computation methods for different values of the defocus parameter $f$ we plot in Figure 7 the values of the
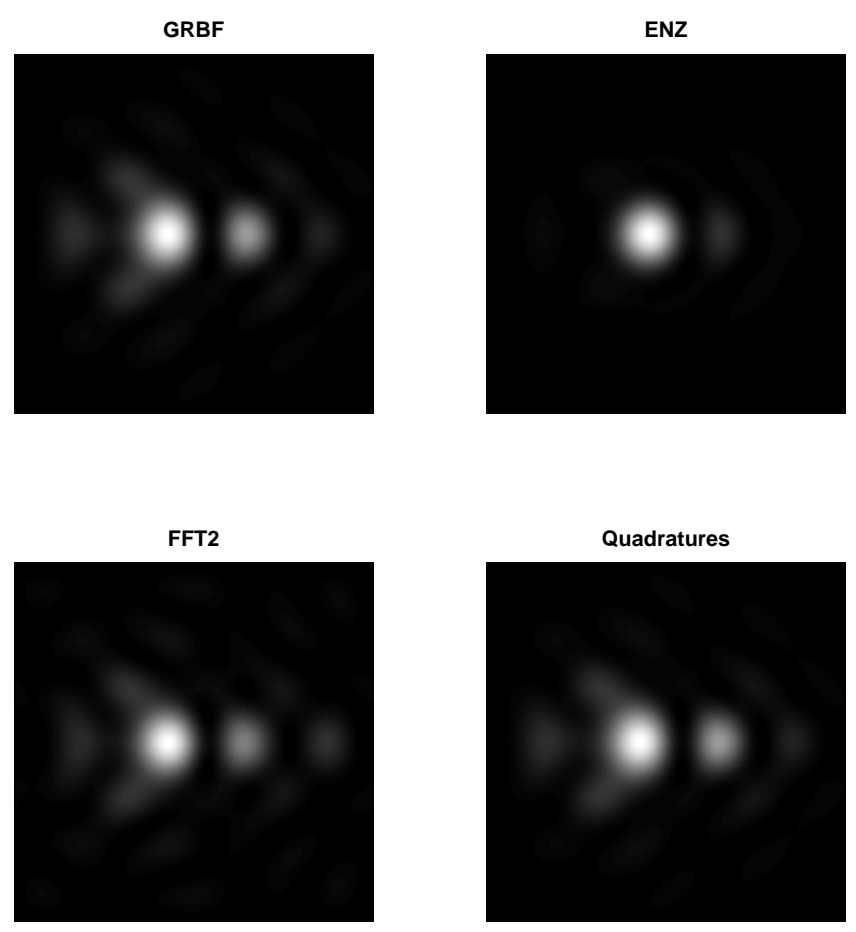

Fig. 5. PSF of the wavefront (11) with $f=0$, calculated by each method. In the first row, from left to right, using GRBF and ENZ. Second row: by FFT2 and quadrature.
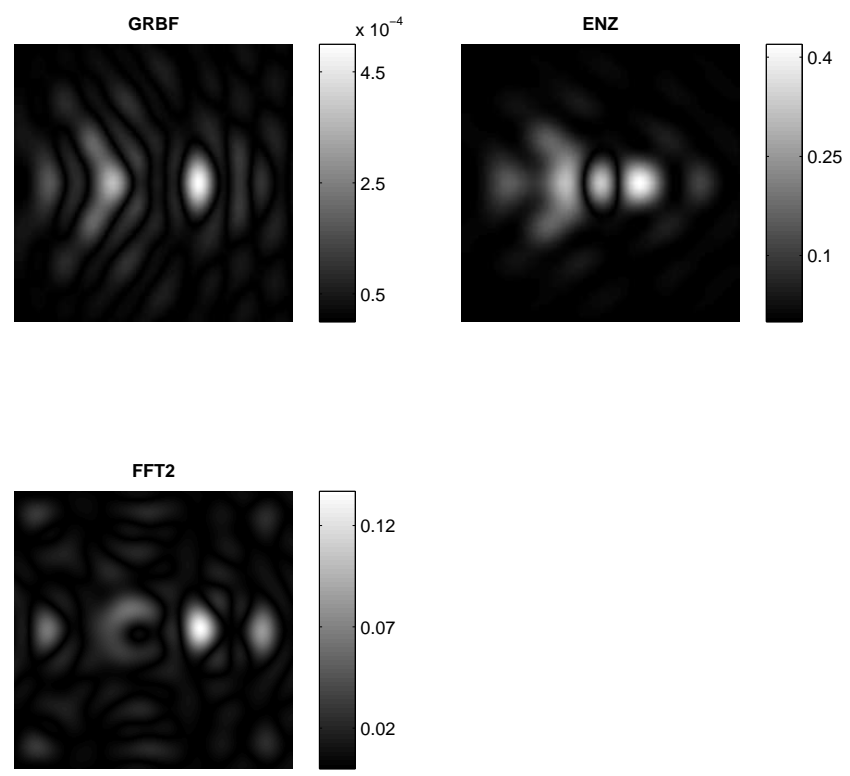

Fig. 6. Absolute error in the PSFs of Figure 5 for each method, with respect to the solution by quadratures.

normalized PSF along the horizontal line $(\phi=0, \pi$ and $r \in[0,1])$.

As a partial conclusion we see that for the simulated wavefront (11) the GRBF method outperforms the other two in several significant digits. 

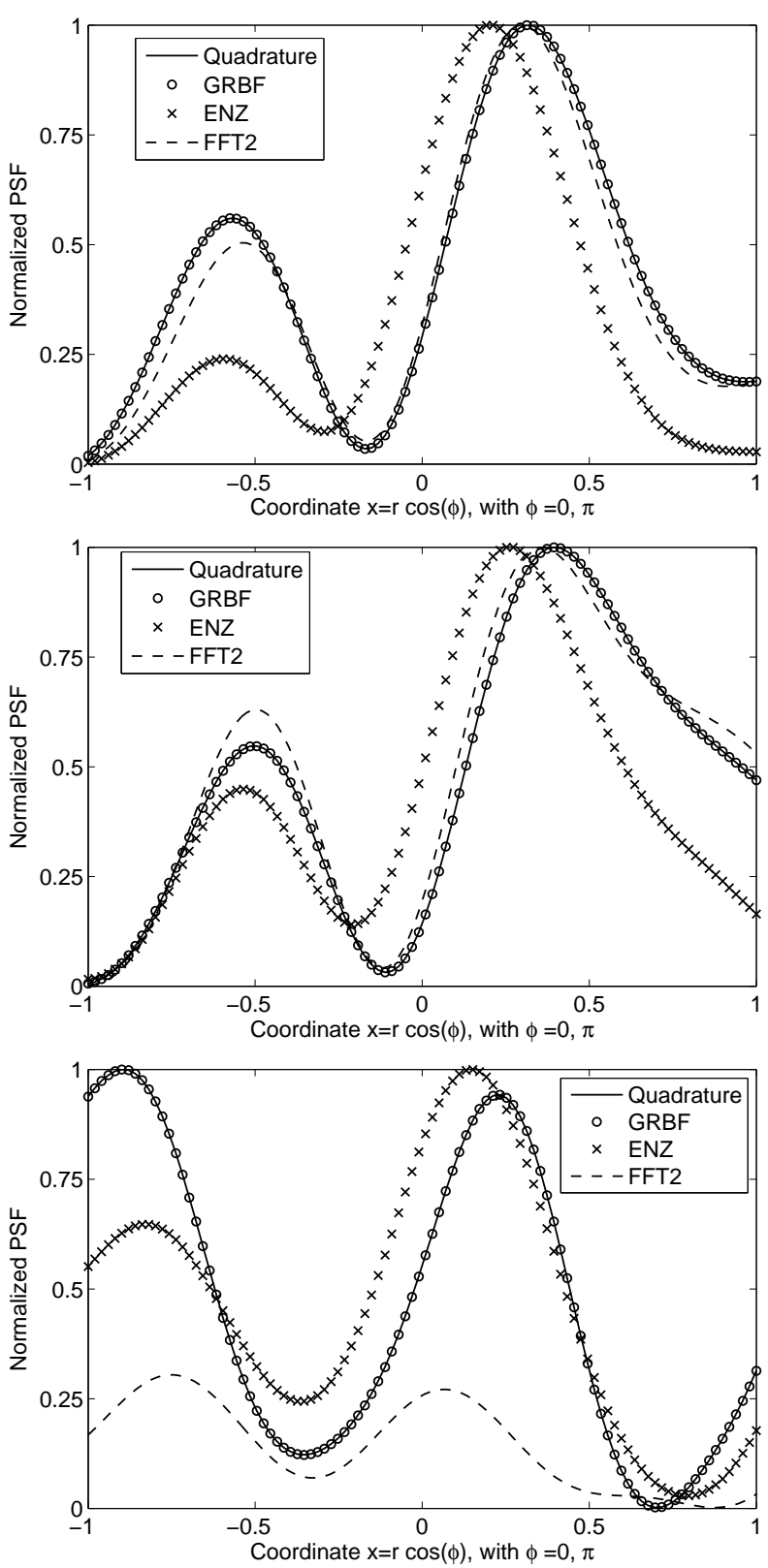

Fig. 7. Values of the normalized PSFs of Figure 5 along the horizontal diameter of the unit disk, calculated for each method, for $f=1$ (top), $f=3$ (middle) and $f=10$ (bottom).

\section{CONCLUSIONS}

A new formula for computing the diffraction integral with variable defocus has been developed. The proposed approach has been compared with the two existing procedures, i.e., the bi-dimensional fast Fourier transform and the extended Nijboer-Zernike theory. The former is a standard numerical procedure, and the latter provides also analytical formulas to evaluate the diffraction integral. The results of the comparison show that the new scheme is very competitive, providing higher accuracy and speed. The main advantage of the new approach is that the increase of the computational cost for a vector of values of the defocus parameter is practically negligible, providing a substantial increase in the performance with respect to the other techniques. This is a reliable and efficient way of obtaining the through-focus characteristics of the eye at higher resolutions in reasonable time.

Additionally, the GRBF approach allows for a straightforward implementation of the multi-resolution scheme. Since each function used for approximation of the pupil function enters the final expression linearly, and taking into account the computational speed of the method, one can use two or more layers of GRBF to fit the residual error consecutively using different sets of centers and different shape parameters in order to improve the accuracy of the results. This idea being currently tested is beyond the scope of this paper and will be discussed in a future paper.

In the virtual refraction paradigm $[12,13]$, the proposed GRBF approach allows calculating through-focus characteristic of the human eye at a very low computational cost for an arbitrarily selected set of the defocus parameters, making it particularly attractive in the studies of dynamic wavefront aberrometry and accommodation.

\section{APPENDIX A: OBTAINING THE PARAMETERS IN} (3)

The goal is expressing the complex pupil functions as a linear combination of GRBF functions, to obtain a expression of the type of (3). Suppose that the wavefront values are known in a discrete and finite set of points at the unit disk (if they are in a bigger disk, they may be rescaled easily). Hence, we have the data set $\left(x_{j}, y_{j}, w_{j}\right)$, $j=1, \ldots, M$, with $w_{j}=W\left(x_{j}, y_{j}\right)$. Values $w_{j}$ can be directly measured or obtained from another set of functions, such as the Zernike polynomials.

\section{Choosing the basis}

A GRBF can be expressed in Cartesian coordinates as $g_{k}(x, y)=\exp \left\{-L_{k}\left[\left(x-a_{k}\right)^{2}+\left(y-b_{k}\right)^{2}\right]\right\}$, where $\left(a_{k}, b_{k}\right)$ are the Cartesian coordinates of its center, and $L_{k}>0$ is its shape parameter, which sets the "scale" of the function $g_{k}$ (and is directly related to the variance of the Gaussian distribution). Transforming it to polar coordinates yields the set of basis functions

$$
g_{k}(\rho, \theta)=\exp \left\{-L_{k}\left(q_{k}^{2}+\rho^{2}-2 \rho q_{k} \cos \left(\theta-\alpha_{k}\right)\right)\right\},
$$

with $\left(q_{k}, \alpha_{k}\right)$ being the polar coordinates of the corresponding centers; $a_{k}=q_{k} \cos \left(\alpha_{k}\right)$ and $b_{k}=q_{k} \sin \left(\alpha_{k}\right)$. Thus, we fix this basis, $\left\{g_{k}\right\}_{k=1}^{N}$, choosing the parameters $a_{k}, b_{k}$ and $L_{k}$, or equivalently, $q_{k}, \alpha_{k}$ and $L_{k}$, for each $1 \leq k \leq N$.

Choosing the same shape parameter $L>0$ for all basis functions simplifies the computations greatly, so we will follow this convention, which can be modified for a multi-resolution scheme, as explained above. We can use 
some statistical criteria for its selection, such as crossvalidation, or even trial and error [33]. In practice, a value between 1 and 20 is satisfactory. Then, taking $N=r^{2}$, we create a grid of $r \times r$ GRBF centers covering the unit disk. Usually, a regular grid on the square $[-1.2,1.2]^{2}$ yields good results. This square covers the unit disk and some peripheral area, which can smooth the Gibb's phenomenon at the boundary of the disk.

\section{Computing the coefficients}

For a fixed set of GRBF functions the purpose is to compute coefficients $c_{k}$ in such a way that their linear combination reproduces the complex pupil function

$$
\sum_{k=1}^{N} c_{k} g_{k}\left(x_{j}, y_{j}\right) \approx P\left(x_{j}, y_{j}\right)=A\left(x_{j}, y_{j}\right) \exp \left\{i w_{j}\right\} .
$$

Notice that the fitting procedure is in practice unavoidable regardless the used approach. It should be pointed out however that the problem of computation of $U$ is ill-conditioned: the values of $U$ are highly sensitive to small oscillations in $\Phi$, so the quality of approximation of the pupil function is critical. The standard procedure is the linear least-squares fit (with complex values), see e.g. [34].

This idea can actually be applied to any set of functions, so the complex pupil function can be expressed in a similar way as a linear combination of other basis functions (for instance, the Zernike polynomials).

\section{APPENDIX B: DERIVATION OF (9)}

Expression (2) shows that $U$ is linear for $P$, so that it is sufficient to find an analytic expression for $U$ when the pupil function is

$$
P(\rho, \theta)=\exp \left\{-L\left(q^{2}+\rho^{2}-2 \rho q \cos (\theta-\alpha)\right)\right\} .
$$

From a direct substitution in (2) we see that we need to calculate integrals of the form

$$
I=\int_{0}^{2 \pi} \exp (2 L \rho q \cos (\theta-\alpha)) \exp (2 \pi i \rho r \cos (\theta-\phi)) d \theta,
$$

which can be evaluated analytically:

$$
I=2 \pi I_{0}(2 \sqrt{\Omega} \rho)
$$

where $I_{0}$ is the modified Bessel function [32, Ch. 10], and

$$
\Omega=L_{k}^{2} q_{k}^{2}+2 \pi i r L_{k} q_{k} \cos \left(\phi-\alpha_{k}\right)-\pi^{2} r^{2} .
$$

It remains to use the series expansion for $I_{0}$ to arrive at (5)-(6), and in consequence, at (9).

\section{ACKNOWLEDGEMENTS}

The first two authors (DRL and AMF) have been supported in part by the research project MTM2011-28952C02-01 from the Ministry of Science and Innovation of Spain and the European Regional Development Fund (ERDF), by Junta de Andalucía, Research Group FQM229 and the Excellence Grant P11-FQM-7276, and by Campus de Excelencia Internacional del Mar (CEIMAR) of the University of Almería. Additionally, AMF was supported by the Excellence Grant P09-FQM-4643 from Junta de Andalucía, and DRL was supported by the FPU program of the Ministry of Education of Spain.

\section{References}

[1] A. Artal, S. Marcos, I. Miranda, and M. Ferro, "Through focus image quality of eyes implanted with monofocal and multifocal intraocular lenses," Opt. Eng. 34, 772779 (1995).

[2] S. Marcos, S. Barbero, and I. Jimenez-Alfaro, "Optical quality and depth-of-field of eyes implanted with spherical and aspheric intraocular lenses," J. Refract. Surg. 21, 1-13 (2005).

[3] P. A. Piers, H. A. Weeber, P. Artal, and S. Norrby, "Theoretical comparison of aberration-correcting customized and aspheric intraocular lenses," J. Refract. Surg. 23, 374-384 (2007).

[4] S. Marcos, E. Moreno, and R. Navarro, "The depthof-field of the human eye from objective and subjective measurements," Vis. Res. 39, 2039-2049 (1999).

[5] B. Wang and K. J. Ciuffreda, "Depth-of-focus of the human eye: Theory and clinical implications," Survey Ophthal. 51, 75-84 (2006).

[6] D. A. Atchison, "Depth of focus of the human eye," in Presbyopia: Origins, effects and treatment, I. Pallikaris, S. Plainis, and W. N. Charman, eds. (Slack Incorporated, 2012).

[7] A. J. Lang, V. Lakshminarayanan, and V. Portney, "Phenomenological model for interpreting the clinical significance of the in vitro optical transfer function", J. Opt. Soc. Am. A 10, 1600-1610 (1993).

[8] A. Guirao, J. Porter, D. R. Williams, and I. G. Cox, "Calculated impact of higher-order monochromatic aberrations on retinal image quality in a population of human eyes: erratum", J. Opt. Soc. Am. A 19, 620-628 (2002).

[9] L. Llorente, S. Barbero, J. Merayo, and S. Marcos, "Total and corneal optical aberrations induced by laser in situ keratomileusis for hyperopia", J. Refract. Surg. 20, 203-216 (2004).

[10] M. J. Collins, T. Buehren, and D. R. Iskander, "Retinal image quality, reading and myopia", Vis. Res. 46, 196215 (2006).

[11] B. Vasudevan, J. K. Ciuffreda and B. Wang, "Subjective and objective depth-of-focus", J. Mod. Opt. 54, 13071316 (2007).

[12] L. N. Thibos, X. Hong, A. Bradley, and R. A. Applegate, "Accuracy and precision of objective refraction from wavefront aberrations", J. Vis. 4, 329-361 (2004).

[13] X. Cheng, A. Bradley, and L. N. Thibos, "Predicting subjective judgment of best focus with objective image quality metrics", J. Vis. 4, 310-321 (2004). 
[14] G. E. Legge, K. T. Mullen, G. C. Woo, and F. W. Campbell, "Tolerance to visual defocus", J. Opt. Soc. Am. A 4, 851-863 (1987).

[15] N. M Jansonius and A. C. Kooijman, "The effect of spherical and other aberrations upon the modulation transfer of the defocused human eye", Ophthal. Physiol. Opt. 18, 504-513 (1998).

[16] F. Yi, D. R. Iskander, and M. J. Collins, "Estimation of the depth of focus from wavefront measurements", J. Vis. 10, 4:3 (2010).

[17] L. N. Thibos, X. Hong, A. Bradley and X. Cheng, "Statistical variation of aberration structure and image quality in a normal population of healthy eyes", J. Opt. Soc. Am. A 19, 2329-2348 (2002).

[18] J. Tarrant, A. Roorda, and C. F. Wildsoet, "Determining the accommodative response from wavefront aberrations", J. Vis. 10, 5:4 (2010).

[19] D. R. Iskander, B. A. Davis, M. J. Collins, and R Franklin, "Objective refraction from monochromatic wavefront aberrations via Zernike power polynomials", Ophthal. Physiol. Opt. 27, 245-255 (2007).

[20] J. Nam, L. N. Thibos, and D. R. Iskander, "Zernike radial slope polynomials for wavefront reconstruction and refraction", J. Opt. Soc. Am. A 26, 1035-1048 (2009).

[21] A. R. FitzGerrell, E. R. Dowski, and W. T. Cathey, "Defocus transfer function for circularly symmetric pupils", Appl. Opt. 36, 5796-5804 (1997).

[22] J. Schwiegerling, "Analysis of the optical Performance of Presbyopia Treatments with the defocus transfer function", J. Refract. Surg. 23, 965-971 (2007).

[23] J. Schwiegerling and J. Choi, "Application of the polychromatic defocus transfer function to multifocal lenses", J. Refract. Surg. 24, 965-969 (2008).

[24] A. J. E. M. Janssen, "Extended Nijboer-Zernike approach for the computation of optical point-spread functions", J. Opt. Soc. Am. A 19, 849-857 (2002).

[25] J. J. M. Braat, P. Dirksen, and A. J. E. M. Janssen, "Assessment of an extended Nijboer-Zernike approach for the computation of optical point-spread functions", J. Opt. Soc. Am. A 19, 858-870 (2002).

[26] A. Martínez-Finkelshtein, A.M. Delgado, G.M. CastroLuna, A. Zarzo, and J.L. Alió. "Comparative analysis of some modal reconstruction methods of the shape of the cornea from corneal elevation data", Invest. Ophtalmol. Vis. Sci. 50, 5639-5645 (2009).

[27] A. Martínez-Finkelshtein, D. Ramos-López, G.M. Castro-Luna, and J.L. Alió. "Adaptive corneal modeling from keratometric data", Invest. Ophtalmol. Vis. Sci. 52, 4963-4970 (2011).

[28] M. Montoya-Hernández, M. Servín, D. MalacaraHernández and G. Paez. "Wavefront fitting using Gaussian functions". Opt. Comm. 163, 259-269 (1999).

[29] D. H. Bailey, and P. N. Swartztrauber. "A fast method for the numerical evaluation of continuous Fourier and Laplace transforms", SIAM J. Sci. Comp. 15, Vol. 5, 1105-1110 (1994).

[30] E. Candès, L. Demanet, and L. Ying. "A fast butterfly algorithm for the computation of Fourier integral operators", Multiscale Model. Simul. 7, Vol. 4, 1727-1750 (2009).

[31] M. Gai, and R. Cancelliere. "An efficient point spread function construction method", Mon. Not. R. Astron. Soc. 377, 1337-1342 (2007).

[32] NIST handbook of mathematical functions. Edited by F. W. J. Olver, D. W. Lozier, R. F. Boisvert and Ch. W. Clark. National Institute of Standards and Technology, Washington, DC; Cambridge University Press, Cambridge, 2010.

[33] G. E. Fasshauer. Meshfree approximation methods with Matlab (World Scientific, 2007).

[34] P. Ch. Hansen, V. Pereyra, and G. Scherer, Least Squares Data Fitting with applications (J. Hopkins Univ. Press, 2013). 